\title{
A 52-Year-Old Man With Vertigo: A Common Symptom or an Urgent Syndrome?
}

\author{
Christina Flourou ${ }^{\mathrm{a}, \mathrm{c}}$, Andreas Tofarides ${ }^{\mathrm{a}}$, Konstantinos Parperis ${ }^{\mathrm{b}}$
}

\begin{abstract}
Basilar occlusion syndrome is an uncommon cause of posterior circulation cerebrovascular syndromes. Approximately one-fifth of the ischemic strokes occur in the posterior cerebrovascular system and it is associated with an increased morbidity and mortality. The symptoms and signs are non-specific, resulting in delays in diagnosis, and a high index of suspicion is required that will lead to the correct diagnosis. Herein, we present a case of a 52-year-old patient who presented to the emergency department with acute onset of dizziness and vertigo due to partial thrombosis of the basilar artery.
\end{abstract}

Keywords: Basilar occlusion syndrome; Central vertigo; HINTS

\section{Introduction}

Basilar artery occlusion syndrome is a subset of posterior circulation cerebral infarctions and represents $1-4 \%$ of all ischemic strokes [1]. Due to the extensive vascular supply of the vertebrobasilar system, the clinical presentation varies between mild symptoms such as dizziness, vertigo, extremity weakness, dysarthria to severe motor deficits. This case highlights the importance of the clinical examination to distinguish central from peripheral vertigo.

\section{Case Report}

A 52-year-old man, smoker, with a past medical history of hypertension, presented to the emergency department with acute onset of dizziness and vertigo gradually worsening over the past $12 \mathrm{~h}$. The patient had moderate relief with oral dimenhy-

Manuscript submitted June 10, 2020, accepted June 20, 2020

Published online June 29, 2020

anternal Medicine Department, Nicosia General Hospital, 2029 Nicosia, Cyprus

${ }^{b}$ University of Cyprus, Medical School, 2029 Nicosia, Cyprus

${ }^{\mathrm{c} C o r r e s p o n d i n g ~ A u t h o r: ~ C h r i s t i n a ~ F l o u r o u, ~ I n t e r n a l ~ M e d i c i n e ~ D e p a r t m e n t, ~}$ Nicosia General Hospital, 2029 Nicosia, Cyprus.

Email: christinafl@hotmail.gr

doi: https://doi.org/10.14740/jmc3525 drinate. He denied similar episodes in the past.

On physical examination, the blood pressure was 150/95 $\mathrm{mm} \mathrm{Hg}$ and the heart rate was $75 \mathrm{bpm}$. On the neurological examination, the patient was alert, oriented with unremarkable cranial nerve, motor and sensory function exam. However, on the cerebellar examination, the Romberg and Unterberger signs were positive. Bidirectional and horizontal nystagmus in primary gaze was evident and the head impulse nystagmus test of skew (HINTS) showed deviation of the eyes. Audiometry and vestibular tests were normal.

Given the presence of bilateral nystagmus, the abnormal HINTS test and the normal vestibular testing, benign paroxysmal positional vertigo and Meniere disease were unlikely diagnoses. Computed tomography of the brain and angiography (CTA) were performed and showed basilar artery partial thrombosis $7 \mathrm{~mm}$ peripheral to the posterior cerebral arteries (Fig. 1).

The patient presented $12 \mathrm{~h}$ after the onset of symptoms, therefore, intravenous thrombolysis was not an appropriate option, and the endovascular mechanical thrombectomy was the initial therapeutic approach. During his hospitalization, the patient had complete resolution of the dizziness and vertigo, and magnetic resonance imaging (MRI) of the brain, angiography and digital subtraction angiography (DSA) demonstrated successful revascularization of the posterior cerebral arteries (Fig. 2).

\section{Discussion}

Basilar artery occlusion syndrome represents a subset of the posterior circulation cerebrovascular syndromes, and may present with a wide range of acute neurologic symptoms and signs [2]. The posterior cerebral circulation comprises of the vertebral arteries that join the basilar artery to form the vertebrobasilar system, and at the base of the brain both carotid and vertebrobasilar system form the circle of Willis [3]. The clinical presentation varies between patients and it is determined by the site of involvement, including the cerebra, medulla, midbrain and occipital cortex. Patients may present with a wide range of symptoms, from mild transient ischemic events, for example, cranial nerve palsy to ischemic strokes with severe motor deficits, such as tetraplegia or a locked-in state.

The most common etiologies and risk factors include atherosclerotic disease, embolism, traumatic dissection of the artery, and less often, arteritis due to Behcet's [3]. Risk factors are similar to those of ischemic strokes, such as hypertension, diabetes mellitus, hyperlipidemia, history of smoking and pro- 


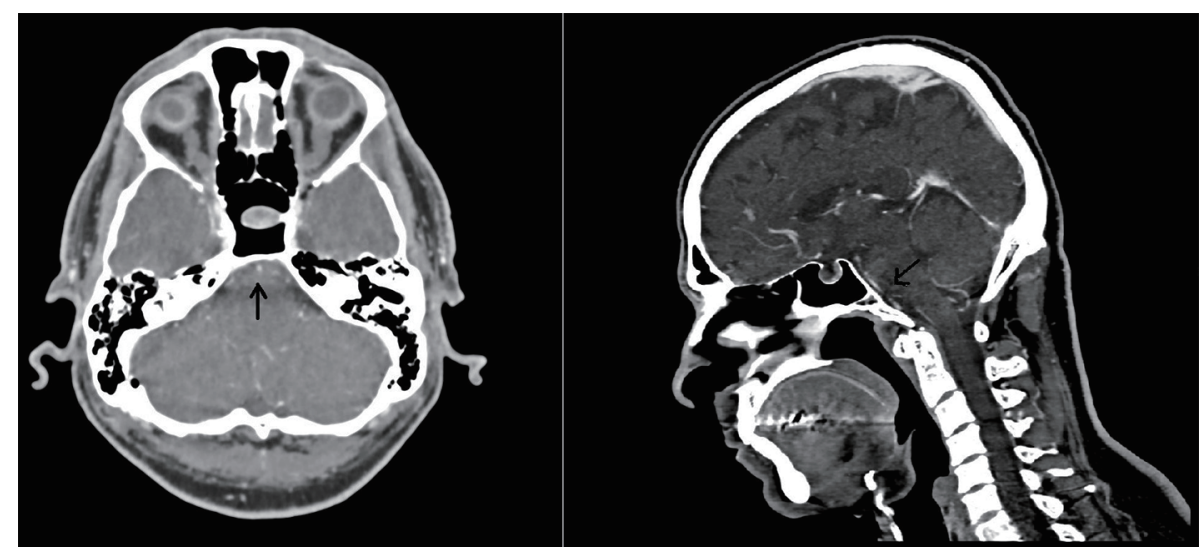

Figure 1. Computed tomography angiography showing basilar artery sub-occlusion $0.7 \mathrm{~cm}$ peripheral to the upper cerebral arteries.

coagulant conditions associated with thrombophilia. HINTS is a sensitive tool to distinguish the central from peripheral vertigo and positive results should be further evaluated with imaging studies. Vertical skew and bidirectional nystagmus is highly suggestive of a central origin of the sign. Head impulse test is normal when the eyes remain fixed upon their targets with no corrective eye movements. Imaging studies can determine the location of the vascular lesion and the appropriate therapeutic approach.

Computed tomography/angiography (CT/CTA) or diffuseweighted MRI/MRA are the appropriate diagnostic studies, although false negative results can occur the first $48 \mathrm{~h}$ after the onset of symptoms. MRA is considered a more sensitive diagnostic modality for the identification of occlusion [4]. Catheter angiography is an invasive procedure, so noninvasive imaging is recommended. The treatment approach includes systemic thrombolysis, and more recently, mechanical endovascular thrombectomy is an additional therapeutic option. In this case, the only therapeutic option beyond the 6-h time window was the mechanical endovascular thrombectomy as demonstrated by a previous trial for ischemic stroke caused by a large artery occlusion of posterior circulation [5].

Posterior circulation cerebral infarctions should be included in the neurological differential diagnosis of central vertigo. Clinical evaluation tools such as HINTS are useful in distinguishing between central to peripheral vertigo. The clinical response to $\mathrm{H} 1$ antagonists does not exclude central vertigo. Clinical evaluation tools are necessary for prompt diagnosis and early therapeutic interventions with thrombolysis or thrombectomy.

\section{Acknowledgments}

None to declare.

\section{Financial Disclosure}

None to declare.
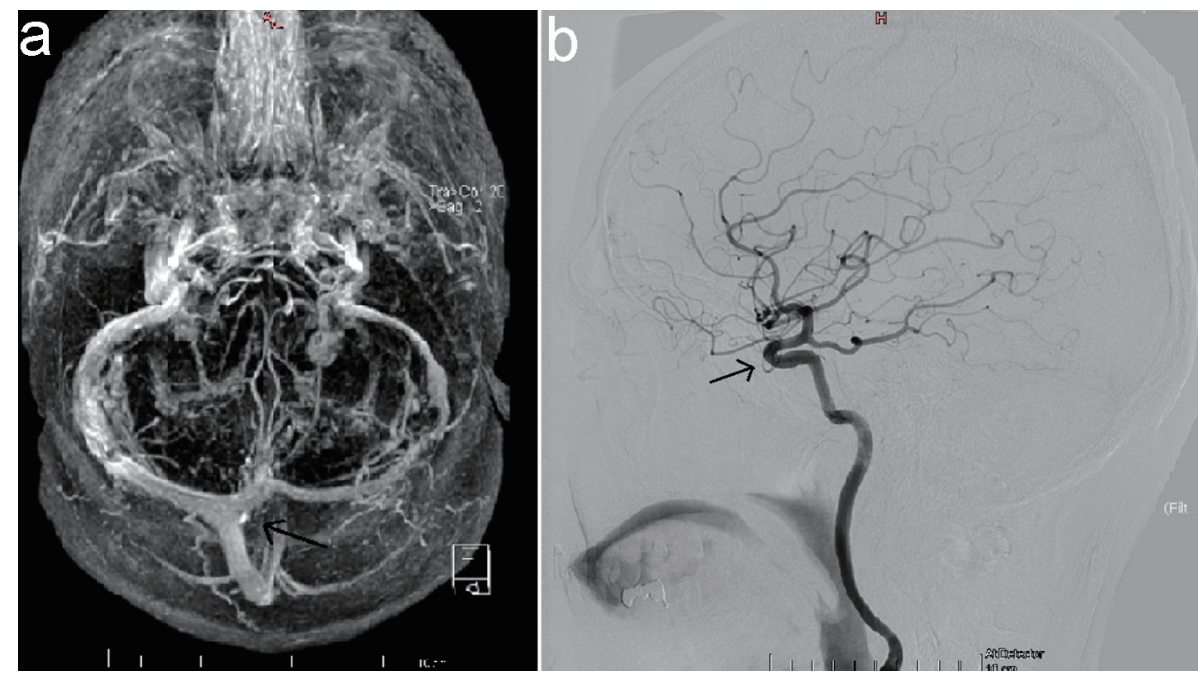

Figure 2. Magnetic resonance imaging of the brain angiography (a) and digital subtraction angiography of the brain (b) demonstrating successful revascularization of the posterior cerebral arteries. 


\section{Conflict of Interest}

None to declare.

\section{Informed Consent}

The patient gave his informed consent.

\section{Author Contributions}

$\mathrm{CF}$ and $\mathrm{AT}$ wrote the paper. KP revised the paper.

\section{Data Availability}

Any inquiries regarding supporting data availability of this study should be directed to the corresponding author.

\section{References}

1. Sarraj A, Medrek S, Albright K, Martin-Schild S, Bibars W, Vahidy F, Grotta JC, et al. Posterior circulation stroke is associated with prolonged door-to-needle time. Int J Stroke. 2015;10(5):672-678.

2. Lindsberg PJ, Mattle HP. Therapy of basilar artery occlusion: a systematic analysis comparing intra-arterial and intravenous thrombolysis. Stroke. 2006;37(3):922928.

3. Oshima K, Sakaura H, Iwasaki M, Nakura A, Fujii R, Yoshikawa H. Repeated vertebrobasilar thromboembolism in a patient with severe upper cervical instability because of rheumatoid arthritis. Spine J. 2011;11(2):e15.

4. Safdarian M, Rohani M. Basilar artery thrombosis on brain MRI. Neurohospitalist. 2018;8(3):NP3.

5. Mogueira RG, Jadhav AP, Haussen DC, et al. Thrombectomy 6 to 24 hours after stroke with a Mismatch between Deficit AND Infarct. N Engl J Med. 2018;378:1. 